\title{
Anisotropy in fission fragment and prompt neutron angular distributions
}

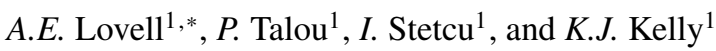 \\ ${ }^{1}$ Los Alamos National Laboratory, Los Alamos, New Mexico, 87545, USA
}

\begin{abstract}
Several physics mechanisms can lead to the deviation from an isotropic angular distribution for both fission fragments and the neutrons that are emitted during the fission event. Two of these effects have recently been implemented into CGMF, the Monte Carlo fission event generator developed at Los Alamos National Laboratory: angular distribution sampling for fission fragments and pre-equilibrium neutrons (those emitted before the compound nucleus forms). Using these new developments, we show that the anisotropy of the neutrons reflects the anisotropy of the fission fragments, in particular as the outgoing energy of neutrons increases. Correlations between the fission fragment and neutron anisotropies could be used to extract the fission fragment anisotropy from the neutron angular distributions.
\end{abstract}

\section{Introduction}

Accurately describing the fission process is important for many applications from the formation of the compound nucleus to the emission of prompt and $\beta$-delayed neutrons and $\gamma$ rays. A few codes are available to calculate the emission of neutrons and $\gamma$ rays during fission events, either on an event-by-event basis or through direct calculations of the prompt neutron and $\gamma$-ray observables [1-6]. For event-by-event models used to study correlations between fission observables, it is also important to be able to describe the angular distributions of the fission fragments and emitted particles. The opening angle between pairs of neutrons or neutrons and fission fragments can be calculated and compared with experimental data, e.g. [7] and references therein.

In this work, we focus on the angular distributions of the fission fragments and prompt neutrons. There are several mechanisms that could cause these angular distributions to be anisotropic. For neutron-induced fission, momentum conservation between the incident neutron and the fissile target boosts the fragments and emitted particles along the beam axis in the forward direction. The fission fragments are also emitted in a preferential direction with respect to the beam axis [8] that depends on the incident neutron energy and is due to the fission transition states that are populated at scission. The angular distribution of the pre-equilibrium neutrons (emitted before a compound nucleus is formed) is forwarded peaked [9], compared to the other isotropically emitted pre-fission neutrons. In addition, neutrons emitted from the fission fragments could have a non-isotropic angular distribution from the spin of the fission fragments or could be emitted from partially accelerated fragments - instead of fully accelerated fragments - which would cause these angular distributions to be anisotropic in the lab

\footnotetext{
*e-mail: lovell@lanl.gov
} 
frame. Attempts have been made to describe the emission of neutrons from accelerating fission fragments, e.g. [10], and models such as the Time-Dependent Superfluid Local Density Approximation (TDSLDA) [11] could provide useful insights into this question. While work is ongoing to experimentally investigate whether neutrons are emitted from fully accelerated fragments or not, most often, the discrepancy between modeled and experimental neutron angular distributions are attributed to scission neutrons-although the percentage of neutrons emitted in this manner is still somewhat of a controversy [12-16]. In this work, we focus on the effects of kinematics, fission fragment anisotropies, and pre-equilibrium neutron angular distributions. While the kinematics of the beam have always been taken into account, the latter two effects have recently been implemented into the fission event generator developed at Los Alamos National Laboratory (LANL), CGMF [1, 2], and here, we show the effects on the fission fragment and neutron anisotropies when these models are included.

\section{Theory}

In this section, we briefly describe the theories behind the fission fragment anisotropies and pre-equilibrium neutron emission. We will also give some details about the CGMF code.

\subsection{Fission fragment angular distributions}

Angular anisotropies of fission fragments as a function of incident neutron energy have been measured for several fissile targets [17-27] and have been shown to change as the incident energy increases. It was postulated [8] that this preferential direction of fragment emission comes from the transition states in the compound nucleus that are populated at the outer saddle point.

The angular anisotropy is defined as a ratio of the cross section at $0^{\circ}$ to the cross section at $90^{\circ}, W\left(0^{\circ}\right) / W\left(90^{\circ}\right)$. The angular distributions can be described by [28],

$$
W(\theta)=\sum_{s(J, K, M)} P_{s}(J, K, M) \frac{2 J+1}{2}\left|d_{M, K}^{J}(\theta)\right|,
$$

where $P_{s}(J, K, M)$ is the population of each state, $s$, identified by the total angular momentum of the compound nucleus, $J$, its projection onto the fission axis, $K$, and its projection onto the beam axis, $M$. The angular dependence is encoded in the Wigner $d$-matrices, $d_{M, K}^{J}(\theta)$.

\subsection{Pre-equilibrium neutron angular distributions}

At high enough incident neutron energies, pre-fission neutrons can be emitted before the compound nucleus forms, i.e. pre-equilibrium neutrons. The energy spectrum and angular distribution of these neutrons differ from the other pre-fission neutrons that are emitted from a compound nucleus. These neutrons can be described by the quantum mechanical theory of Feshbach, Kerman, and Koonin (FKK) [29], in which the angular distribution can be separated into a multi-step direct and multi-step compound part. The pre-equilibrium neutrons are described by the multi-step direct piece of this formulation and are similar to inelastically scattered neutrons.

Recently, the angular distribution of pre-equilibrium neutrons was extracted experimentally for the first time for ${ }^{239} \mathrm{Pu}(n, f)$ [30]. The pre-equilibrium channel opens around $E_{\text {inc }}=12 \mathrm{MeV}$, and the neutron angular distributions are significantly forward peaked. These angular distributions are well-described by the FKK theory and can also be reproduced by Kalbach systematics [31]. 


\subsection{CGMF}

CGMF is a Monte Carlo Hauser-Feshbach code developed at LANL to describe the evaporation of prompt neutrons and $\gamma$ rays emitted from fission fragments [1,2]. The required inputs are scission-fragment yields in mass, charge, total kinetic energy, spin, and parity, $Y(A, Z, T K E, J, \pi)$. The mass yields, $Y(A)$, are three-Gaussian fits to experimental data $[32,33]$, and the charge yields, $Y(Z \mid A)$, are taken from Wahl systematics [34]. The TKE distribution for each mass, $Y(T K E \mid A)$, is also taken to be Gaussian. The spin of each fragment is sampled from $Y(J)=(J+1) \exp \left(-\alpha J(J+1) / 2 B^{2}\right)$ where $B^{2}$ depends on the nuclear temperature and moment of inertia and $\alpha$ is a global scaling parameter [35]. The parity is evenly sampled, either +1 or -1 .

Once the initial conditions are sampled, the total excitation energy, TXE, is determined from the $Q$-value, TXE $=Q-$ TKE. The excitation energy is then shared between the two fragments based on a ratio, $R_{T}$, which is fitted as a function of mass to reproduce the average neutron multiplicity, $\bar{v}(A)$. From the excitation energy of each fragment, neutrons and $\gamma$ rays are evaporated probabilistically through the Hauser-Feshbach statistical theory. The neutron probabilities are calculated through transmission coefficients using an optical potential parameterization (Koning-Delaroche, by default), and the $\gamma$-ray probabilities are determined from the $\gamma$-ray strength function for E1, M1, and E2 transitions. The fissions are calculated on an event-by-event basis, and for each event, the initial conditions of the fission fragments are recorded along with the momenta and energies of the fragments, neutrons, and $\gamma$ rays. In this way, both average and correlated quantities can be constructed.

\section{Fitting procedure}

In this section, we describe the process for fitting and sampling the fission fragment anisotropies and pre-equilibrium angular distributions, along with describing how the fragment and neutron anisotropies are extracted from the CGMF calculations.

\subsection{Fission fragments}

For most of the fissile targets considered in CGMF, there are several sets of experimental data across the incident neutron energy range of interest, from thermal to $20 \mathrm{MeV}$. For each target, we fit the available data using cubic splines. One spline fit compared to experimental data is shown in Fig. 1 for ${ }^{239} \mathrm{Pu}(n, f)$. There is good coverage of the ${ }^{239} \mathrm{Pu}(n, f)$ data across the whole energy range, and with only a few knots, we are able to reproduce the fluctuations in the fission fragment anisotropy.

The angular dependence in $\cos \theta$ of the light fragment is sampled from the distribution,

$$
P(\cos \theta) \propto 1+\left(\frac{W\left(0^{\circ}\right)}{W\left(90^{\circ}\right)}-1\right) \cos ^{2} \theta .
$$

When $W\left(0^{\circ}\right) / W\left(90^{\circ}\right)=1, \cos \theta$ is sampled isotropically. In all cases, $\phi$ is sampled isotropically. The direction of the heavy fragment is constructed through momentum conservation in the center-of-mass frame of the compound nucleus.

Relying on a cubic spline fit does limit our inclusion of the fission fragment anisotropy to isotopes where data have been measured over a wide range of incident energies. For ${ }^{241} \mathrm{Pu}(n, f)$, data are only available up to $E_{\text {inc }}=8 \mathrm{MeV}$. In this case, the data over this range have the same shape and magnitude as the anisotropy for ${ }^{239} \mathrm{Pu}(n, f)$ so we are able to use the same fit as a function of incident neutron energy. Using a spline fit implicitly accounts for 


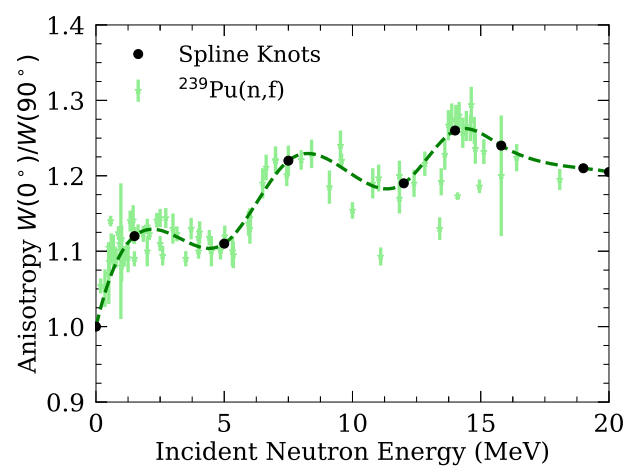

Figure 1. Fission fragment anisotropy as a function of incident neutron energy for ${ }^{239} \mathrm{Pu}(n, f)$. Data is shown as the green points, spline knots as black filled circles, and the cubic spline fit as the dashed dark green line.

multi-chance fission about the neutron separation energy which makes this approach only an approximation. In principle, Eq. (1) could be used to construct these angular distributions, the weights $P_{s}(J, K, M)$ are unknown and are often fitted to the measured angular distributions, instead of predicted directly from theory.

\subsection{Pre-equilibrium neutrons}

Angular distributions for the pre-equilibrium neutrons were calculated for ${ }^{239} \mathrm{Pu}$ using the quantum mechanical theory of Feshbach, Kerman, and Koonin [29] over a range of incident neutron energies and target excitation energies [36]. This calculation gives an approximate shape for the angular distributions due to the limited model space. To smooth out any irregularities and to have a parameterization across all incident and excitation energies, the angular distributions were fitted using Kalbach systematics [31],

$$
\frac{d \sigma}{d \Omega}=a\left(E_{\mathrm{inc}}, E^{*}\right) \sinh (\cos \theta)+b\left(E_{\mathrm{inc}}, E^{*}\right)
$$

where $E_{\mathrm{inc}}$ is the incident neutron energy and $E^{*}$ is the excitation energy of the target system. The parameters $a$ and $b$ are taken to be polynomial in $E_{\text {inc }}$ and $E^{*}$. Although these calculations were performed for ${ }^{239} \mathrm{Pu}$, the same fits were used for all targets within CGMF as the addition or removal of a few nucleons does not significantly change the calculated angular distributions.

\subsection{Extracting the angular anisotropy}

To extract the angular anisotropy from the CGMF calculations, we bin the calculated $\cos \theta$ values then fit these angular distributions to a sum of Legendre polynomials,

$$
W(\theta)=p_{0} L_{0}(\cos \theta)+p_{1} L_{1}(\cos \theta)+p_{2} L_{2}(\cos \theta)
$$

where $p_{0}, p_{1}$, and $p_{2}$ are the fitting parameters weighing each of the Legendre polynomials. Since the fission fragment angles are sampled from a $\cos ^{2} \theta$ distribution, we should not need any higher order terms to describe these angular distributions. The $L_{1}(\cos \theta)$ linear term is included to take into account the boost of the fragments from the center-of-mass frame to the 
laboratory frame. The three Legendre polynomial parameterization of Eq. (4) is used to fit the angular distribution of the neutrons as well.

We use this fitting procedure instead of directly comparing the number of counts at $0^{\circ}$ and $90^{\circ}$ to mitigate effects of low statistics on the mean values of the anisotropy, in particular when we begin to consider the angular distributions of neutrons with an increasing energy threshold, $E_{\text {thres }}$. This fitting procedure also allows us to extract uncertainties on the resulting anisotropy values. The covariance between the best-fit parameters of Eq. (4) can be constructed. A distribution of parameter sets can then be calculated by sampling a multi-variate Gaussian distribution centered around the best-fit parameter values with a variance given by the constructed covariance matrix. These parameter sets can then each be used to calculate the anisotropy from Eq. (4). Two thousand anisotropy values are calculated for each energy of interest, and $95 \%$ confidence bands are constructed by removing the most outlying $5 \%$ of the calculations.

\section{Results}

We can first look at the changes to the fission fragment and neutron anisotropies when kinematics, fission fragment anisotropy, and pre-equilibrium angular distributions are included into CGMF. The effects of kinematics had already been implemented into CGMF. The anisotropy of the fission fragments, neutrons emitted from the fission fragments, and all neutrons (from the fission fragments and the pre-fission process) from just the recoil is shown in Fig. 2a. Here, the anisotropy of the fission fragments is less than $5 \%$ at $E_{\text {inc }}=20 \mathrm{MeV}$, and as the neutrons are emitted either isotropically from the fission fragments or from the target nucleus, the anisotropy of the neutrons is lower. Figure $2 \mathrm{~b}$ shows the anisotropies after the fission fragment anisotropy is taken into account, and c shows the anisotropies when the forward-peaked pre-equilibrium angular distributions are sampled. In Fig. 2b, there is little difference between the anisotropies of the neutrons when pre-fission neutrons are or are not considered, except due to the slight differences in the kinematics of the compound after a prefission neutrons is emitted. When the angular distribution of the pre-equilibrium neutrons is taken into account, there is a noticeable increase in the overall anisotropy of all neutrons.

In each case, the neutron anisotropy tracks the fission fragment anisotropy but is overall lower in magnitude, as the angular distributions are flatter compared to those of the fission fragments. Also, when the pre-equilibrium neutron angular distributions are sampled, we see very little change in the fission fragment anisotropy and the anisotropy of the neutrons emitted directly from the fission fragments. Above about $E_{\text {inc }}=12 \mathrm{MeV}$, the anisotropy increases when all of the neutrons are considered, where the pre-equilibrium neutrons are first emitted. Their forward-peaked angular distributions are clearly reflected in the resulting neutron anisotropy, even though they account for less than $20 \%$ of the emitted pre-fission neutrons.

As the outgoing neutron threshold energy, $E_{\mathrm{thres}}$, increases, the neutron angular distributions - and thus the neutron anisotropy - tends toward that of the fission fragments. In Fig. 3 , we show the neutron anisotropy as a function of incident neutron energy compared to the measured fission fragment anisotropy for a the neutrons emitted from the fission fragments and $b$ when all emitted neutrons are taken into account. As the neutron threshold energy increases, the neutron anisotropy more closely tracks the fission fragment anisotropy, but the uncertainty on the extracted anisotropy also increases. This is due to statistics; the higher energy neutrons are more rare than the low-energy neutrons, and the fitting procedure of the Legendre polynomials becomes more uncertain.

From Fig. 3b, we also see that including the pre-fission neutrons in this analysis complicates the picture. At the highest incident energies, the neutron anisotropies are larger than 

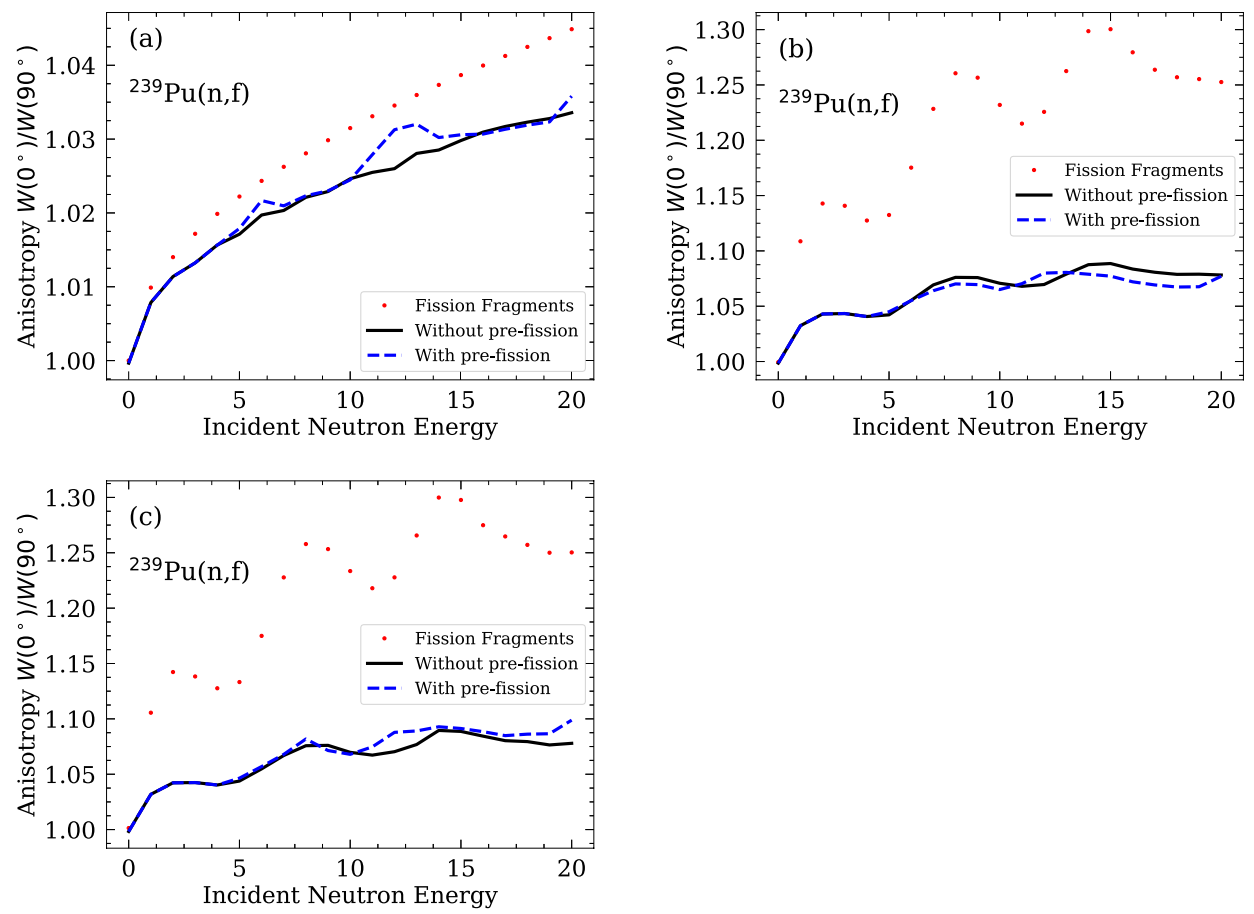

Figure 2. Comparison of the anisotropy for fission fragments, neutrons emitted only from the fragments, and all neutrons emitted during the fission reaction for ${ }^{239} \mathrm{Pu}(n, f)$ when (a) only recoil is included (fission fragments and pre-equilibrium neutrons are sampled isotropically), (b) recoil and the fission fragment anisotropy are included, and (c) recoil, fission fragment anisotropy, and pre-equilibrium neutron angular distribution sampling are included.
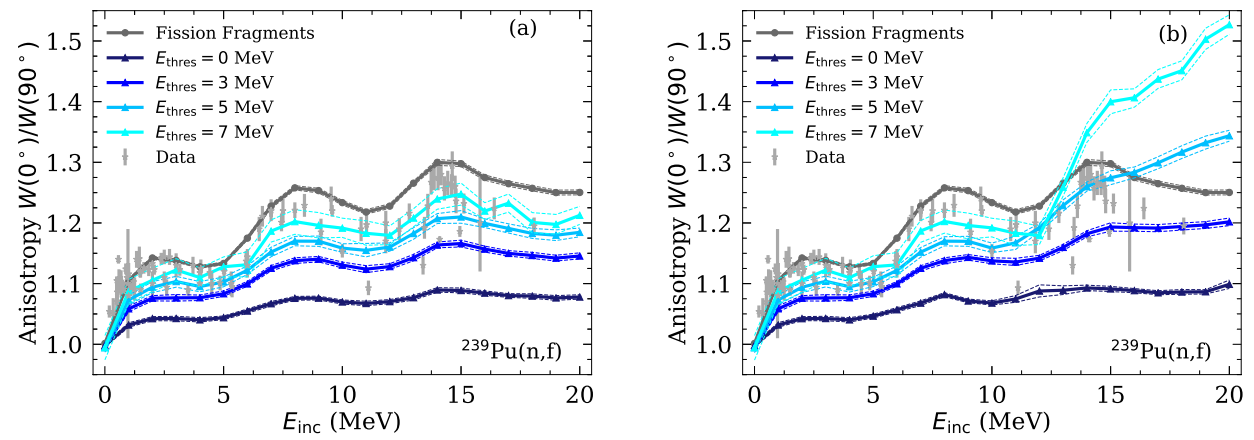

Figure 3. Neutron anisotropy as a function of incident neutron energy for $E_{\text {thres }}=0,3,5$, and $7 \mathrm{MeV}$ (in indigo, blue, turquoise, and cyan, respectively) compared to experimentally measured fission fragment anisotropy (grey stars) for (a) neutrons emitted from the fission fragments and (b) all neutrons emitted during the fission event for ${ }^{239} \mathrm{Pu}(n, f)$. Also for comparison, the fission fragment anisotropy calculated from CGMF in dark grey.

the fission fragment anisotropies and become increasingly so as the threshold energy rises. However, as the threshold energy rises, the incident energy where the pre-equilibrium neutrons have an effect also increases. The increase in the anisotropy caused by the pre-fission 
neutrons is pushed to higher incident energies. Therefore, the incident energy to which the fission fragment anisotropy can be extracted from the neutron anisotropy increases if the neutron threshold energy can be increased.

There is, of course, a trade-off between the neutron threshold energy and the statistics. Fewer neutrons are emitted with higher outgoing energies. For codes such as CGMF, these challenges can be mitigated by running longer calculations. Collecting more statistics experimentally is a larger effort. However, for a given incident energy and threshold energy, a correction factor could be deduced from CGMF to convert the neutron anisotropy to the fission fragment anisotropy.

\section{Conclusion}

In this work, we have newly included two sources of anisotropy into the fission decay code CGMF: the angular anisotropy of the fission fragments coming from the transition states that are populated at the outer saddle point and of the pre-equilibrium neutrons. In addition to the angular anisotropies that are induced by the recoil of the target due to the incident neutron, which had already been taken into account in CGMF, these effects lead to clear angular anisotropies in both the fission fragments and the prompt neutrons that are emitted. These effects have now been taken into account in CGMF for neutron-induced fission on ${ }^{233,234,235,238} \mathrm{U}$, ${ }^{237} \mathrm{~Np}$, and ${ }^{239,241} \mathrm{Pu}$.

To include the fission fragment anisotropy, experimental data was fit with cubic splines and the fission fragment angles were sampled from a $\cos ^{2} \theta$ distribution. Pre-equilibrium neutron angular distributions were calculated across a range of incident energies and excitation energies using the Feshbach, Kernan, and Koonin quantum mechanical model and fit globally to Kalbach systematics. The anisotropy of the prompt neutrons reflects the anisotropy of the fission fragments as a function of incident neutron energy, although the neutron anisotropy is significantly lower. By removing lower energy neutrons from the analysis, the magnitude of the neutron anisotropy comes closer to that of the fission fragments. However, these events occur less frequently, and the analysis begins to suffer from lack of statistics. Still, by utilizing the correlations between the fission fragment and neutron anisotropies seen in CGMF, the fission fragment anisotropy could be extracted from a measurement of the neutron anisotropy.

This work was performed under the auspice of the U.S. Department of Energy by Los Alamos National Laboratory under Contract 89233218 CNA000001 and was supported by the Office of Defense Nuclear Nonproliferation Research \& Development (DNN R\&D), National Nuclear Security Administration, U.S. Department of Energy. We gratefully acknowledge the support of the U.S. Department of Energy through the LANL/LDRD Program and the Center for Non Linear Studies.

\section{References}

[1] P. Talou, I. Stetcu, P. Jaffke, M. Rising, A. Lovell, T. Kawano, Comput. Phys. Commun. 269, 108087 (2021)

[2] B. Becker, P. Talou, T. Kawano, Y. Danon, I. Stetcu, Phys. Rev. C 87, 014617 (2013)

[3] J. Verbeke, J. Randrup, R. Vogt, Computer Physics Communications 191, 178 (2015)

[4] J. Verbeke, J. Randrup, R. Vogt, Computer Physics Communications 222, 263 (2018)

[5] S. Okumura, T. Kawano, P. Talou, P. Jaffke, S. Chiba, J. Nucl. Sci. Tech. 55, 1009 (2018)

[6] O. Litaize, O. Serot, L. Berge, The European Physical Journal A 51, 177 (2015)

[7] P. Talou, R. Vogt, J. Randrup, M.E. Rising, S.A. Pozzi, J. Verbeke, M.T. Andrews, S.D. Clarke, P. Jaffke, M. Jandel et al., The European Physical Journal A 54, 9 (2018) 
[8] A. Bohr, On the Theory of Nuclear Fission, in International Conference on the Peaceful Uses of Atomic Energy, edited by N.Y. United Nations, Vol. 2 (Geneva, 1956), p. 151

[9] E. Gadioli, P.E. Hodgson, Pre-Equilibrium Nuclear Reactions (Oxford Scientific Publications, 1992)

[10] A. Matsumoto, H. Taninaka, K. Hashimoto, T. Ohsawa, Journal of Nuclear Science and Technology 49, 782 (2012), https://doi.org/10.1080/00223131.2012.677127

[11] A. Bulgac, et al., in progress (2021)

[12] H.R. Bowman, S.G. Thompson, J.C.D. Milton, W.J. Swiatecki, Phys. Rev. 126, 2120 (1962)

[13] H.R. Bowman, J.C.D. Milton, S.G. Thompson, W.J. Swiatecki, Phys. Rev. 129, 2133 (1963)

[14] S.S. Kapoor, R. Ramanna, P.N.R. Rao, Phys. Rev. 131, 283 (1963)

[15] K. Skarsvåg, K. Bergheim, Nuclear Physics 45, 72 (1963)

[16] N. Carjan, M. Rizea, Phys. Rev. C 99, 034613 (2019)

[17] A.S. Vorobyev, A.M. Gagarski, O.A. Shcherbakov, L.A. Vaishnene, A.L. Barabanov, JETP Letters 102, 203 (2015)

[18] S. Ahmad, M.M. Islam, A.H. Khan, M. Khaliquzzaman, M. Husain, M.A. Rahman, Nuclear Science and Engineering 71, 208 (1979)

[19] J.E. Brolley, W.C. Dickinson, R.L. Henkel, Phys. Rev. 99, 159 (1955)

[20] J.E. Simmons, R.L. Henkel, Phys. Rev. 120, 198 (1960)

[21] V. Geppert-Kleinrath, F. Tovesson, J.S. Barrett, N.S. Bowden, J. Bundgaard, R.J. Casperson, D.A. Cebra, T. Classen, M. Cunningham, D.L. Duke et al. (NIFFTE Collaboration 2), Phys. Rev. C 99, 064619 (2019)

[22] R.L. Henkel, J.E. Brolley, Phys. Rev. 103, 1292 (1956)

[23] E. Birgersson, A. Oberstedt, S. Oberstedt, F.J. Hambsch, Nuclear Physics 817, 1 (2009)

[24] I. Ryzhov, M. Onegin, G. Tutin, J. Blomgren, N. Olsson, A. Prokofiev, P.U. Renberg, Nuclear Physics 760, 19 (2005)

[25] F. Vivès, F.J. Hambsch, H. Bax, S. Oberstedt, Nuclear Physics 662, 63 (2000)

[26] R.B. Leachman, L. Blumberg, Phys. Rev. 137, B814 (1965)

[27] L. Blumberg, R.B. Leachman, Phys. Rev. 116, 102 (1959)

[28] C. Wagemans, The Nuclear Fission Process (CRC Press, 1991)

[29] H. Feshbach, A. Kerman, S. Koonin, Annals of Physics 125, 429 (1980)

[30] K.J. Kelly, T. Kawano, J.M. O’Donnell, J.A. Gomez, M. Devlin, D. Neudecker, P. Talou, A.E. Lovell, M.C. White, R.C. Haight et al., Phys. Rev. Lett. 122, 072503 (2019)

[31] C. Kalbach, Phys. Rev. C 37, 2350 (1988)

[32] I. Stetcu, P. Talou, T. Kawano, EPJ Web Conf. 146, 04026 (2017)

[33] I. Stetcu, P. Talou, T. Kawano, EPJ Web of Conferences 122, 01012 (2016)

[34] A.C. Wahl, Tech. rep., Los Alamos Nat. Lab. LA-13928 (2002)

[35] I. Stetcu, P. Talou, T. Kawano, M. Jandel, Phys. Rev. C 90, 024617 (2014)

[36] T. Kawano, private communication (2018) 